\title{
Binary White Dwarfs in the Galactic Halo
}

\section{Pim van Oirschot ${ }^{1, *}$, Gijs Nelemans ${ }^{1,2}$, Amina Helmi ${ }^{3}$, Else Starkenburg, ${ }^{4}$, Onno Pols ${ }^{1}$ and Anthony G.A. Brown ${ }^{5}$}

${ }^{1}$ Department of Astrophysics / IMAPP, Radboud University Nijmegen, P.O. Box 9010, 6500 GL Nijmegen, The Netherlands

*email: P.vanOirschot@astro.ru.nl

${ }^{2}$ Institute for Astronomy, KU Leuven, Celestijnenlaan 200D, 3001 Leuven, Belgium

${ }^{3}$ Kapteyn Astronomical Institute, University of Groningen, P.O. Box 800, 9700 AV, Groningen, The Netherlands

${ }^{4}$ Department of Physics and Astronomy, University of Victoria, P.O. Box 3055, STN CSC, Victoria BC V8W 3P6, Canada

${ }^{5}$ Leiden Observatory, Leiden University, P.O. Box 9513, 2300 RA Leiden, The Netherlands

We use the stellar population synthesis code SeBa (Portegies Zwart \& Verbunt (1996), Toonen, Nelemans \& Portegies Zwart (2012)) to study the halo white dwarf population. Here we assume a Kroupa initial mass function and compare 4 models, varying two parameters: the star formation (SF) history of the halo (either continuous SF during 2.5 Gyr, which started 13.2 Gyr ago, or a SF burst during $360 \mathrm{Myr}$, which started $12.9 \mathrm{Gyr}$ ago - see the left panel of the figure) and the binary fraction of the halo (either $100 \%$ single stars, or $100 \%$ binaries). White dwarf cooling models (Althaus et al. (2009) and Renedo et al. (2010)) allow us to plot the halo white dwarf luminosity function for these 4 models, as is done in the right panel of the figure. Combined with an assumption about the density distribution of halo stars, we will study which of these white dwarfs Gaia can see, and what that can tell us about the initial parameter distributions in the halo. In the near future, we plan to use the Munich-Groningen semi-analytical galaxy formation model (Starkenburg et al. (2013)), to obtain key ingredients for the population synthesis modeling, such as a realistic star formation history (see the left panel of the figure).
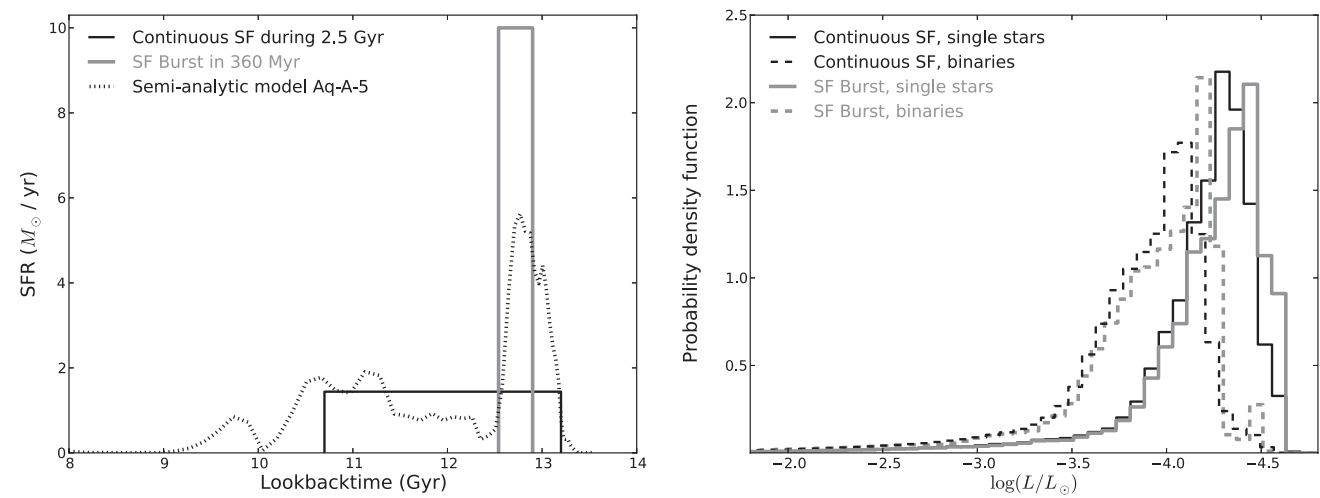

\section{References}

Portegies Zwart, S. \& Verbunt, F. 1996, A\& A, 309, 179

Toonen S., Nelemans, G., \& Portegies Zwart, S. 2012, A\& A, 546, A70

Althaus, L. G., et al. 2009, A\& $A, 502,207$

Renedo, I., et al. 2010, ApJ, 717, 183

Starkenburg, E., et al. 2013, MNRAS, 429, 725 